\title{
«WHITE CITY, BLACK CITY» DE SHARON ROTBARD
}

\author{
Antoine Mandoux ${ }^{1}$
}

\begin{abstract}
"[...] in the problematic political contexts and difficult circumstances of any possible architectural practice anywhere, and especially in Israel, writing has always seemed to me one of the few decent and effective ways to be an architect." p. 184.

"It is not really necessary to state again that the White City will never be white enough - the earth simply does not rotate fast enough.»p. 177.
\end{abstract}

En 2004, la «Ville blanche de Tel Aviv » est classée au patrimoine mondial de l'UNESCO parce qu'elle est «la synthèse d'une valeur exceptionnelle des diverses tendances du Mouvement moderne en matière d'architecture et d'urbanisme au début du $\mathrm{XX}^{\mathrm{e}}$ siècle. » Le discours officiel qui a soutenu l'élection de la ville lie intimement la naissance et la croissance de Tel Aviv au Bauhaus et au Style International apportés par les premiers «migrants » juifs venus d'Europe centrale. Si on le suit un peu plus loin, les fondements de la ville reposent sur des dunes de sable blanc, apportant modernité et prospérité à une terre délaissée par ses anciens habitants. Avec "White City/Black City ", Sharon Rotbard, architecte israélien, commence une critique systématique de cette véritable hagiographie de l'édification de Tel Aviv à nos jours.

Usant de faits historiques et politiques comme d'éléments de la culture populaire, Sharon Rotbard compose morceau à morceau une histoire de l'histoire, le récit du récit dans lequel l'architecture devient un moyen d'écrire la narration et la narration de construire la ville. Concevoir une ville n'est pas un acte anodin et la forme qu' elle prend reflète les valeurs et les aspirations de ses concepteurs et, dans le même mouvement, ces valeurs et aspirations, une fois matérialisés, conditionnent ceux qui la vivent. De cette manière, la conception de Tel Aviv est un projet politique qui relève plus des visées coloniales sionistes et britanniques que de tout autre mythe fondateur.

Ne recensant que peu d'édifices construits, encore moins d'architectes israéliens, qui auraient pu être liés au mouvement Bauhaus, le livre révèle que la ville dans sa structure comme dans sa forme doit bien plus au Mandat britannique qu'à tout autre pouvoir en place, même le nouvellement indépendant Etat hébreu. De ce point de vue, Tel Aviv ressemble plus à Casablanca ou Alger, des ensembles urbains

1 Étudiant Erasmus, Master Population, Société et Territoire, Instituto de Geografia e Ordenamento do Território da Universidade de Lisboa. (U.L.B.) Compte rendu préparé pour le Séminaire Géopolitique et Mondialisation. E-mail: amandoux@ campus.ul.pt 
modernistes et « européanisés » élaborés par le colonisateur français qu'au projet national ou nationaliste qu'il est censé être. Le pouvoir colonial britannique est le premier - et peut-être le seul - à avoir envisagé la cité et sa région comme un tout et à les avoir redessinées selon leurs intérêts : traçant les axes liant l'arrière-pays au port commercial de Jaffa, ébauchant les structures d'un état moderne, détruisant, divisant ou développant des quartiers urbains pour éviter les révoltes et les troubles. Comme nous le voyons, l'histoire de Tel Aviv est un peu plus complexe que le conte officiel et Sharon Rotbard nous propose dans son style brillant et acerbe de passer de l'autre côté du miroir et de rencontrer la « Ville noire »- encore aujourd'hui les parties les plus sombres et pauvres de la ville.

Si la «Ville blanche » repose sur des dunes, la « Ville noire » a été bâtie sur de florissants vergers d'agrumes et les restes de la ville de Jaffa, les uns et les autres ayant fait la renommée de la région.

Dès lors, commence le récit passionnant de l'histoire tragique de Jaffa, autrefois l'un des ports les plus prospères de la côte levantine. Du siège sanguinaire par les troupes françaises en 1799 à sa capture et son annexion par le tout neuf Etat hébreu en 1948, l'auteur retrace dans le détail près d'un siècle et demi d'une histoire tumultueuse sur fond de colonialismes britannique et sioniste et de nationalismes arabe et juif. Des premières colonies au début du $\mathrm{XX}^{\mathrm{e}}$ siècle au projet Homa Umigdali, il expose le mouvement d'expansion et d'accaparement du territoire par les nouveaux arrivants, mouvement qui isolera petit à petit Jaffa de son arrière-pays et qui mènera à ce qu'il nomme son « urbicide ». Si Tel Avivii incarne l'aspiration des populations juives à l'indépendance, Jaffa, de par sa position enclavée par le tracé des frontières de novembre 1947, devient le symbole de la cause palestinienne. Des groupes paramilitaires sionistes, Etzel entre autres, se lancent à l'attaque de la ville déjà partiellement détruite et dont une partie de la population a quitté les lieux et ils l'achèveront, elle et ses derniers habitants, alors même que l'indépendance d'Israël est prononcée.

Après son annexion au territoire israélien et à la municipalité de Tel Aviv, l'« urbicide » de Jaffa continue en s'attaquant à sa mémoire et à sa matérialité. Le champ de ruines qu'elle est devenue est surnommé « The Big Zone » et sert d'accueil aux nouveaux venus arrivés des pays de l'Est et des pays arabes environnants. Ceux-ci, souvent pauvres, lui donnent une mauvaise réputation, à la fois lieux de tous les trafics et de toutes les libertés. Lâchée aux mains de spéculateurs dans les années 60 , le front de mer se hérisse d'hôtels et d'immeubles, des pans entiers de l'ancienne ville sont rasés et reconstruits avec de nouveaux noms, de nouvelles formes et de nouvelles histoires. La nouvelle gare routière est peut-être le signe par lequel la démesure de l'entreprise peut être aperçue : "objet » monolithique d'architecture moderniste dont l'utilité reste encore à prouver. Le musée Etzel est lui aussi un exemple, paradoxe de la politique israélienne : un cube en verre ultra-moderne sur les restes de l'une des dernières maisons jaffoites encore debout et abritant le musée à la gloire des bourreaux de l'ancienne ville arabe...

Si des voix s'élèvent au début des années 80 pour défendre et protéger la «Ville blanche », c'est également pour glorifier le progressisme et l'abnégation des premiers colons, souvent issus de familles allemandes ou d'Europe occidentale, porteuses d'un sioniste, socialiste et athée et dont elle serait l'œuvre ; et ce, après la victoire de la droite israélienne aux élections de 1977, partisane d'une colonisation à tout-va et d'un laisser-aller urbanistique et spéculatif dont la "Ville noire » serait le résultat. Si en 2004, la «Ville blanche » est reconnue internationalement pour son architecture moderne, la "Ville noire » est l'objet d'une vaste opération sécuritaire qui aboutira à l'expulsion de 115.000 travailleurs étrangers, soit autant que la population arabe avant 1947.

Sharon Rotbard, lui-même installé dans la partie « sombre » de la ville, achève son premier livre par une réflexion plus générale sur l'utilisation de l'architecture comme moyen de domination. Reprenant les principales caractéristiques du modernisme - austérité et efficacité, béton et blancheur, il compare leurs usages dans Tel Aviv à d'autres cas de villes neuves comme Dakar, Alger ou Casablanca, 
toutes issues de l'imagination d'urbanistes modernistes et qui portent encore aujourd'hui les formes de la domination coloniale. En plus d'être un exposé intelligent de l'histoire de Tel Aviv-Jaffa selon le prisme de l'urbanisme, ce livre, le premier de Sharon Rotbard, laisse les jalons d'une réflexion plus profonde et dans d'autres contextes de l'emploi de l'architecture comme « arme de guerre».

\section{REFERÊNCIAS BIBLIOGRÁFICAS}

Rotbard, S. (2015). White City, Black City: Architecture and War in Tel Aviv and Jaffa. Cambridge (Massachusetts): MIT Press.

i Voir « Wall and Tower (Homa Umigdal), the mold of israeli architecture » de Sharon Rotbard, in Rafi Segal, Eyal Weizman, "A civilian occupation, the politics of israeli architecture », Babel/Verso, Tel Aviv/London-New York, 2003.

ii Tel Aviv signifie en hébreu « Ancienne-Nouvelle Terre », un autre nom de Eretz Israel, la " Terre promise ». C’est également la traduction du roman de Theodore Herzl, fondateur du sioniste, Altneuland publié en 1902. 